\title{
Association Between Cognitive Function and Health Care Costs 3 Months and 6 Months After Initiating Antidepressant Medication for Depressive Disorders
}

\author{
Valery Walker, MSc; Haridarshan Patel, PharmD; Jonathan L. Kurlander, MS; \\ Breanna Essoi, MPH; Jiao Yang, PhD; Atul R. Mahableshwarkar, MD; \\ Jennifer C. Samp, PharmD, MS; and Kasem S. Akhras, PharmD
}

\begin{abstract}
BACKGROUND: Major depressive disorder is one of the most common and disabling mental health disorders and is associated with substantial costs in terms of direct health care utilization and workplace productivity. Cognitive dysfunction, which alone substantially increases health care costs, is commonly associated with major depressive disorder. However, the health care costs of cognitive dysfunction in the context of depressive disorder are unknown. Recovery from mood symptoms is not always associated with resolution of cognitive dysfunction. Thus, cognitive dysfunction may contribute to health care burden even with successful antidepressant therapy.
\end{abstract}

OBJECTIVE: To compare health care utilization and costs for patients with a depressive disorder with and without cognitive dysfunction, at 3 and 6 months after initiation of antidepressant medication.

METHODS: This was an observational study, combining a cross-sectional patient survey, administered during a telephone interview, with health care claims data from a large, geographically diverse U.S. health plan. Included patients had at least 1 pharmacy claim for an antidepressant medication between August 1 and September 30, 2012, and no claim for any antidepressant during the 6 months prior to the index date. In addition to other criteria assessed in the claims data, patients confirmed a diagnosis of depression or major depressive disorder and the absence of any exclusionary neurological diagnoses possibly associated with cognitive impairment. Eligible patients were administered validated cognitive function assessments of verbal episodic memory (Hopkins Verbal Learning Test-Revised, Delayed and Total); attention (Digit Span Forward Maximum Sequence Length); working memory (Digit Span Backward Maximum Sequence Length); and executive function (D-KEFS-Letter Fluency Test). Based on comparison of scores with normative data, patients were assigned to cognitive dysfunction or cognitive normal cohorts. All-cause (all diagnoses) and depressive disorder-related health care utilization and costs (all from a payer perspective) were assessed 6 months prior (baseline) to antidepressant initiation and 3 months and 6 months after (follow-up) initiation of antidepressant medication. Health care utilization and costs included ambulatory (office and hospital outpatient), emergency room, inpatient hospital, pharmacy, other medical (e.g., laboratory and diagnostics), and total (all categories combined). All-cause and depressive disorder-related total costs during the 3- and 6-month follow-up periods were modeled with generalized linear modeling with gamma distribution and log link, while adjusting for potential confounders (age, race, gender, education, employment, and comorbidities).

RESULTS: Of the 13,537 patients who were mailed an invitation, $824(6 \%)$ were eligible and agreed to participate. Of these, 563 patients provided informed consent, completed the interview, maintained eligibility, and were included in the 3-month calculations. Among these, 255 (45\%) were classified as having cognitive dysfunction. Mean patient age was 41.3 $( \pm 12.5)$ years; $80 \%$ were female. Most patients were white and employed.
More patients in the cognitive normal cohort were white $(P<0.001)$ and employed full time $(P=0.029)$, had higher education attainment $(P<0.001)$, and had fewer comorbidities $(P=0.007)$ than those in the cognitive dysfunction cohort. Over the first 3 months, patients with cognitive dysfunction had higher adjusted all-cause costs $(\$ 3,309$ vs. $\$ 2,157, P=0.002)$ and higher adjusted depressive disorder-related costs ( $\$ 718$ vs. $\$ 406$, $P<0.001)$ than patients without cognitive dysfunction. At 6 months, data from 4 patients were removed from the analysis because of exclusionary diagnoses. Over 6 months, patients with cognitive dysfunction had higher adjusted all-cause costs $(\$ 4,793)$ than patients without cognitive dysfunction $(\$ 3,683, P=0.034)$. Over 6 months, depressive disorder-related costs did not significantly differ between patients with (\$771) and without cognitive dysfunction ( $\$ 594, P=0.071$ ). The main drivers of all-cause costs were office visits, outpatient hospital visits, and inpatient costs, and the main driver of depressive disorder-related costs was inpatient costs.

CONCLUSIONS: Cognitive dysfunction was associated with higher adjusted all-cause and depressive disorder-related costs 3 months after initiation of an antidepressant medication. This difference persisted for all-cause costs through 6 months. Identification and treatment of cognitive dysfunction in patients with depressive disorder might reduce health care costs.

J Manag Care Spec Pharm. 2015;21(9):742-52

Copyright $\odot 2015$, Academy of Managed Care Pharmacy. All rights reserved.

\section{What is already known about this subject}

Major depressive disorder has an estimated lifetime prevalence of 10\%-20\% in the United States and imposes significant economic and societal consequences in terms of direct medical care costs, associated morbidity and mortality, and productivity costs.

Cognitive dysfunction, which is commonly associated with major depressive disorder, may persist even after resolution of other symptoms of depression.

By itself, a diagnosis of cognitive dysfunction is associated with up to triple the health care costs of persons without such a diagnosis. However, costs related to cognitive dysfunction in the context of depression have not been reported.

\section{What this study adds}

Using data from a large U.S. health plan, we analyzed health care utilization and costs for patients with a depressive disorder, with and without cognitive dysfunction, for 3 and 6 months following the initiation of antidepressant medication. 


\section{What this study adds (continued)}

Patients with cognitive dysfunction had significantly more allcause outpatient hospital visits during the first 3 months after initiating antidepressant medication. At 6 months, patients with cognitive dysfunction had significantly more all-cause emergency room visits and depressive disorder-related outpatient visits.

Relative to patients without cognitive dysfunction, patients with cognitive dysfunction had significantly higher all-cause and depressive disorder-related health care costs 3 months following initiation of an antidepressant and higher all-cause costs 6 months following initiation of an antidepressant.

$\mathrm{M}$ ajor depressive disorder (MDD) is one of the most common and disabling mental health disorders, with a lifetime prevalence estimated at 10\%-20\% in the United States and with higher rates for women than men. ${ }^{1-3}$ Depression has significant economic and societal consequences. In 2000, the estimated costs of depression were $\$ 83.1$ billion, including $\$ 26.1$ billion in direct medical costs and $\$ 51.5$ billion in workplace productivity costs. ${ }^{4}$ Per patient annual direct medical costs of depression up to $\$ 2,500$ have been reported. ${ }^{5}$ As a major health problem associated with gradual and often incomplete recovery, MDD imposes significant limitations on functioning and well-being, raises the risk of mortality due to medical illness, and increases utilization of health services and associated costs to society. ${ }^{6}$

Cognitive dysfunction is commonly associated with MDD and is characterized by "difficulty thinking, concentrating, or making decisions," which is part of the MDD diagnostic criteria in the Diagnostic and Statistical Manual of Mental Disorders, 5th edition. ${ }^{7}$ Resolution of depression is not always associated with recovery from cognitive dysfunction, and there is increasing evidence of persistent cognitive dysfunction even after resolution of depressive symptoms. ${ }^{8-10}$ Thus, cognitive dysfunction may contribute to health care burden even after successful treatment of other symptoms of MDD.

Cognitive dysfunction by itself is associated with higher health care costs. In fact, people diagnosed with dementia, an extreme form of cognitive dysfunction, have experienced triple the health care costs of persons with no such diagnosis. ${ }^{11}$ The costs related to cognitive dysfunction in the context of depression have not been reported. The objectives of this study were to compare health care utilization and costs from a payer perspective for patients with a depressive disorder, with and without cognitive dysfunction, for 3 months and 6 months after the start of antidepressant medication. These results will be of importance to health care providers and payers involved with the treatment of patients with a depressive disorder experiencing cognitive dysfunction.

\section{Methods}

\section{Design}

This was a real-world, observational study, combining a crosssectional patient survey administered during a telephone interview with patients' health care claims data from the Optum Research Database (ORD). The ORD contains data for approximately 33.9 million patients with commercial insurance, representing all U.S. geographic census regions. ${ }^{12}$

Pharmacy claims data were used to identify patients initiating an antidepressant medication between August 1 and September 30, 2012 (the date of the first antidepressant pharmacy claim is the index date). In November 2012, identified patients were mailed an invitation to participate in the study. A telephone interview was conducted for each participant between November 14 and December 28, 2012. Cognitive function assessments and other patient-reported outcomes were collected during the telephone interviews. Pharmacy and medical claims data were used to calculate health care costs in the 6-month period pre-index date (baseline period) and the 3- and 6-month periods post-index date (follow-up periods).

\section{Patients}

The study protocol received a waiver of authorization and ethics approval from the New England Institutional Review Board, and all patients completed informed consent and health information release forms. Patients had at least 1 pharmacy claim for an antidepressant between August 1 and September 30, 2012, and no claim for any antidepressant during the 6 months prior to the index date. Medical claims for recent depressive episodes were unavailable at the time of patient identification due to a 6-month lag required for complete medical claims; therefore, each patient confirmed a diagnosis of depression or MDD and the absence of any exclusionary neurological diagnoses possibly associated with cognitive impairment (see Appendices A-D, available in online article, for complete inclusion/exclusion criteria).

\section{Demographics and Clinical Characteristics}

Claims data from the baseline period were used to obtain age, gender, and geographic region; to identify comorbid conditions as defined in the Agency for Healthcare Research and Quality Clinical Classification Software; and to calculate the QuanCharlson Comorbidity Index score based on the International Classification of Diseases, Ninth Revision, Clinical Modification (ICD9-CM) diagnosis codes (a measure of comorbidity burden). ${ }^{13,14}$

Eligible patients interviewed by telephone provided race and ethnicity, employment status, occupation, and highest education level achieved. Patient-reported outcome assessments measuring depression severity, disability, self-reported cognitive functioning, and health-related quality of life were also administered, and these results are reported separately. ${ }^{15}$ 


\section{Assessment of Cognitive Function}

Each patient completed a structured assessment of objectively measured cognitive function measuring 4 domains: verbal episodic memory (Hopkins Verbal Learning Test-Revised [HVLT-R] Delayed and Total); attention (Digit Span Forward [DSF] Maximum Sequence Length); working memory (Digit Span Backward [DSB] Maximum Sequence Length); and executive function (D-KEFS-Letter Fluency Test). ${ }^{16-18}$ These tests were chosen because they represent the domains found to be most impaired in acute depression and have been shown to be sensitive to depression-related cognitive dysfunction. ${ }^{19-21}$ Performance on each test was compared with published ageand gender-stratified normative data and then used to classify each patient's cognition as either normal or dysfunctional. ${ }^{16-18}$ Patients were classified as having "cognitive dysfunction" if they met at least 1 of 2 criteria: (1) two or more test scores 1.5 standard deviations (SD) or more below the normative mean, or (2) three or more test scores 1.0 SD or more below the normative mean. ${ }^{22}$ Patients not meeting either of these criteria were classified as "cognitive normal." See Figure 1 for cohort assignment.

\section{Health Care Utilization and Costs}

Health care utilization and costs from a payer perspective were calculated from pharmacy and medical claims 6 months prior (baseline) to antidepressant initiation, 3 months after initiation of antidepressant medication (3-month follow-up), and 6 months (including the 3-month follow-up) after initiation of antidepressant medication (6-month follow-up). Health care costs represent the sum of health plan and patient-paid amounts and were classified as follows: office visits, outpatient hospital visits, emergency room, inpatient, other medical, pharmacy, and total costs (combining all of these categories). An example of "other medical" costs would be independent laboratory and diagnostic costs. "All-cause" costs included the cost of all health care utilization consumed during the study period, regardless of the diagnosis, and "depressive disorderrelated" costs included medical claims having a primary or nonprimary diagnosis code for, or relating to, depressive disorder (ICD-9-CM codes 296.82 [atypical depressive disorder], 296.2x [major depressive disorder, single episode], 296.3x [major depressive disorder, recurrent episode], 311 [depressive disorder, not elsewhere classified]) and associated pharmacy claims (see Appendices B and E, available in online article).

\section{Statistical Analyses}

Chi-square and t-tests were used to compare demographic characteristics and health care utilization between the cognitive dysfunction and cognitive normal cohorts for categorical and continuous variables, respectively. Two-sided t-tests were used to compare health care costs between the 2 cohorts. We used a generalized linear model (GLM) with gamma distribution and log link to assess the relationship between cognitive function and total all-cause costs, controlling for potential confounders of performance on neuropsychological tests (level of education, race, gender, age, level of employment, Quan-Charlson comorbidity score, and mental health-related comorbidities). Because health care costs are often skewed, estimated cost measures were modeled using Manning and Mullahy's formulation. ${ }^{23}$ This method avoids potential difficulties introduced by transformation and retransformation of the dependent variable. ${ }^{24}$ Coefficients from a GLM are estimated cost ratios.

Adjusted costs for all-cause health care utilization were estimated from the gamma models for 3- and 6-month follow-up periods. The same approach was used to assess the relationship between cognitive function and depressive disorder-related costs, controlling for the same potential confounders.

A logistic regression analysis in which presence of nonzero baseline costs (measured as a binomial) as the dependent variable was performed with a single independent (predictor) variable as presence of cognitive dysfunction (measured as a binomial). Logistic regression assesses the relationship between a binomial (or categorical) dependent variable and a set of independent variables. This model was used because the binomial outcome variable violates assumptions of ordinary least squares regression. A GLM with log link and gamma distribution assessed the relationship between cognitive dysfunction (as a single, binomial predictor variable) and nonzero baseline costs.

\section{Results}

\section{Study Sample}

Of the 13,537 patients who were mailed an invitation to participate in the study, 824 (6\%) were eligible and agreed to participate (Figure 1). Of this number, 563 patients completed the informed consent process, completed a telephone interview, maintained eligibility, and were included in the calculation of 3-month health care utilization and costs. Four patients were removed from the calculation of 6-month costs because they had an exclusionary diagnosis of bipolar disorder during the 6-month follow-up period, leaving 559 patients in the 6-month analysis.

\section{Demographics}

Of the 563 patients in the 3-month follow-up analysis, 255 (45\%) met the criteria for cognitive dysfunction. The mean patient age was $41.3(\mathrm{SD}=12.5)$ years, and $80 \%$ were female Most patients were white and employed. More patients in the cognitive normal cohort were white and employed full time, had higher education, and had fewer comorbidities compared with patients in the cognitive dysfunction cohort. Demographic details are shown in Table 1.

\section{Costs at Baseline}

In the 6 months prior to antidepressant initiation (baseline), the unadjusted all-cause costs were $\$ 6,431$ for the cognitive dysfunction cohort compared with $\$ 3,953$ for the cognitive normal cohort 
TABLE 1 Demographics and Clinical Characteristics by Cohort

\begin{tabular}{|c|c|c|c|c|c|c|c|}
\hline \multirow{2}{*}{$\begin{array}{l}\text { Characteristics } \\
\text { Age, mean (SD), years }\end{array}$} & \multicolumn{2}{|c|}{$\begin{array}{l}\text { Total Sample } \\
\qquad(\mathrm{N}=563)\end{array}$} & \multicolumn{2}{|c|}{$\begin{array}{l}\text { Cognitive Normal } \\
\qquad(n=308)\end{array}$} & \multicolumn{2}{|c|}{$\begin{array}{l}\text { Cognitive Dysfunction } \\
\qquad(\mathrm{n}=255)\end{array}$} & \multirow{2}{*}{$\begin{array}{c}P \text { Value } \\
0.191 \\
\end{array}$} \\
\hline & 41.3 & $(12.5)$ & 41.9 & $(12.5)$ & 40.5 & $(12.5)$ & \\
\hline Female sex, n (\%) & 449 & (79.8) & 250 & $(81.2)$ & 199 & $(78.0)$ & 0.358 \\
\hline \multicolumn{8}{|l|}{ Region, $\mathrm{n}(\%)$} \\
\hline South & 266 & $(47.3)$ & 140 & $(45.5)$ & 126 & $(49.4)$ & \multirow{4}{*}{0.778} \\
\hline Midwest & 184 & $(32.7)$ & 106 & $(34.4)$ & 78 & $(30.6)$ & \\
\hline West & 86 & $(15.3)$ & 47 & $(15.3)$ & 39 & $(15.3)$ & \\
\hline Northeast & 27 & $(4.8)$ & 15 & $(4.9)$ & 12 & $(4.7)$ & \\
\hline \multicolumn{8}{|l|}{ Race, $\mathrm{n}(\%)^{\mathrm{a}}$} \\
\hline White & 516 & $(92.3)$ & 293 & $(95.8)$ & 223 & $(88.1)$ & $<0.001$ \\
\hline Black or African American & 29 & $(5.2)$ & 13 & $(4.3)$ & 16 & $(6.3)$ & 0.271 \\
\hline American Indian & 10 & $(1.8)$ & 6 & $(2.0)$ & 4 & (1.6) & 0.736 \\
\hline Asian & 6 & $(1.1)$ & 3 & $(1.0)$ & 3 & $(1.2)$ & 0.815 \\
\hline Native Hawaiian or other Pacific Islander & 2 & $(0.4)$ & 0 & $(0.0)$ & 2 & $(0.8)$ & 0.119 \\
\hline Other & 12 & $(2.2)$ & 4 & $(1.3)$ & 8 & $(3.2)$ & 0.132 \\
\hline Hispanic/Latino & 36 & $(6.4)$ & 19 & $(6.2)$ & 17 & $(6.7)$ & 0.810 \\
\hline \multicolumn{8}{|l|}{ Employment status, $\mathrm{n}(\%)^{\mathrm{a}}$} \\
\hline Employed full time & 365 & $(64.8)$ & 212 & $(68.8)$ & 153 & $(60.0)$ & 0.029 \\
\hline Employed part time & 105 & $(18.7)$ & 51 & $(16.6)$ & 54 & $(21.2)$ & 0.161 \\
\hline Homemaker & 82 & $(14.6)$ & 48 & $(15.6)$ & 34 & $(13.3)$ & 0.451 \\
\hline Student & 64 & $(11.4)$ & 39 & $(12.7)$ & 25 & $(9.8)$ & 0.288 \\
\hline Unemployed & 43 & $(7.6)$ & 21 & $(6.8)$ & 22 & $(8.6)$ & 0.421 \\
\hline Other & 15 & $(2.7)$ & 11 & (3.6) & 4 & (1.6) & 0.142 \\
\hline Retired & 12 & $(2.1)$ & 7 & $(2.3)$ & 5 & $(2.0)$ & 0.799 \\
\hline \multicolumn{8}{|l|}{ Education, n (\%) } \\
\hline Graduate school & 84 & $(14.9)$ & 60 & $(19.5)$ & 24 & $(9.4)$ & \multirow{7}{*}{$<0.001$} \\
\hline College graduate & 156 & $(27.7)$ & 98 & $(31.8)$ & 58 & $(22.8)$ & \\
\hline Two-year degree & 83 & $(14.7)$ & 46 & $(14.9)$ & 37 & $(14.5)$ & \\
\hline Some college, but no degree & 148 & $(26.3)$ & 72 & $(23.4)$ & 76 & $(29.8)$ & \\
\hline High school or equivalent & 83 & $(14.7)$ & 32 & $(10.4)$ & 51 & $(20.0)$ & \\
\hline Some high school & 8 & $(1.4)$ & 0 & $(0.0)$ & 8 & $(3.1)$ & \\
\hline Less than high school & 1 & $(0.2)$ & 0 & $(0.0)$ & 1 & $(0.4)$ & \\
\hline Quan-Charlson score, mean (SD) & 0.24 & $(0.77)$ & 0.16 & $(0.59)$ & 0.34 & $(0.93)$ & 0.007 \\
\hline Depressive disorder duration, mean (SD), days ${ }^{\mathrm{b}}$ & $2,406.8$ & $(2,790.3)$ & $2,486.8$ & $(2,805.5)$ & $2,304.5$ & $(2,774.7)$ & 0.502 \\
\hline \multicolumn{8}{|l|}{ Time to survey, $\mathrm{n}(\%) \mathrm{c}$} \\
\hline $61-90$ days & 118 & $(21.2)$ & 74 & $(24.3)$ & 44 & $(17.5)$ & \multirow{3}{*}{0.035} \\
\hline 91-120 days & 302 & $(54.3)$ & 151 & $(49.5)$ & 151 & $(60.2)$ & \\
\hline$>120$ days & 136 & $(24.5)$ & 80 & $(26.2)$ & 56 & $(22.3)$ & \\
\hline \multicolumn{8}{|c|}{$\begin{array}{l}\text { aPatients may report more than } 1 \text { category. } \\
\text { bCalculated from patient-reported date of depression diagnosis. } \\
\text { cTime between index antidepressant fill and survey. } \\
\text { SD = standard deviation. }\end{array}$} \\
\hline
\end{tabular}

$(P=0.067$; Table 2). The main cost drivers for all-cause costs were office visits, outpatient hospital visits, and inpatient costs. The mean 6-month baseline unadjusted depressive disorderrelated total costs were $\$ 782$ for the cognitive dysfunction cohort compared with $\$ 108$ for the cognitive normal cohort $(P=0.133)$. The main cost driver for the depressive disorder-related costs in the cognitive dysfunction cohort was inpatient costs of $\$ 702$ for 4 patients with hospitalizations. There were no inpatient costs in the cognitive normal cohort. The results from the multiple logistic regression models comparing the proportion of patients with costs to patients without costs found no significant difference between the 2 cohorts for unadjusted all-cause $(P=0.503)$ or unadjusted depressive disorder-related costs $(P=0.557)$.

The gamma models with a log link that compared costs for all patients, including patients with no costs, found a significant difference between the 2 cohorts for unadjusted allcause $(P<0.001)$ and unadjusted depressive disorder-related costs $(P<0.001)$. When inpatient costs were removed from the gamma model, the difference in unadjusted depressive disorder-related costs between the cohorts was no longer significant. 


\begin{tabular}{|c|c|c|c|c|c|}
\hline Total Costs & $\begin{array}{c}\text { Total Sample } \\
(\$)\end{array}$ & $\begin{array}{l}\text { Cognitive } \\
\text { Dysfunction } \\
(\$)\end{array}$ & $\begin{array}{l}\text { Cognitive } \\
\text { Normal } \\
(\$)\end{array}$ & $\begin{array}{c}\text { Difference } \\
\text { (CD-CN) } \\
(\$)\end{array}$ & $\begin{array}{c}\text { Gamma Model } \\
P \text { Value }\end{array}$ \\
\hline \multicolumn{6}{|l|}{ 6-month baseline } \\
\hline Unadjusted & $\mathrm{n}=564$ & $\mathrm{n}=309$ & $\mathrm{n}=255$ & & \\
\hline All-cause & 5,074 & 6,431 & 3,953 & $2,478^{\mathrm{a}}$ & 0.067 \\
\hline Depressive disorder-related & 412 & 782 & 108 & $674^{a}$ & 0.133 \\
\hline \multicolumn{6}{|l|}{ 3-month follow-up } \\
\hline Unadjusted & $\mathrm{n}=563$ & $\mathrm{n}=308$ & $\mathrm{n}=255$ & & \\
\hline All-cause & 3,053 & 3,948 & 2,312 & 1,636 & 0.113 \\
\hline Depressive disorder-related & 739 & 951 & 564 & 387 & 0.536 \\
\hline Adjusted & & $\mathrm{n}=306$ & $\mathrm{n}=253$ & & \\
\hline All-cause & & 3,309 & 2,157 & 1,152 & 0.002 \\
\hline Depressive disorder-related & & 718 & 406 & 312 & $<0.001$ \\
\hline \multicolumn{6}{|l|}{ 6-month follow-up } \\
\hline Unadjusted & $\mathrm{n}=559$ & $\mathrm{n}=307$ & $\mathrm{n}=252$ & & \\
\hline All-cause & 5,186 & 6,707 & 3,938 & 2,769 & 0.040 \\
\hline Depressive disorder-related & 1,010 & 1,316 & 759 & 557 & 0.387 \\
\hline Adjusted & & $\mathrm{n}=305$ & $\mathrm{n}=250$ & & \\
\hline All-cause & & 4,793 & 3,683 & 1,110 & 0.034 \\
\hline Depressive disorder-related & & 771 & 594 & 177 & 0.071 \\
\hline
\end{tabular}

\section{Follow-up Health Care Utilization}

Table 3 provides the number of patients with at least 1 occurrence of health care utilization as well as the mean number per patient, including those patients with no use of health care utilization. Significantly more patients with cognitive dysfunction than without had all-cause outpatient hospital visits over the first 3 months after antidepressant initiation (32.6\% vs. $24.7 \%, P=0.039$; Table 3). Over 6 months, $46.8 \%$ of patients with cognitive dysfunction had all-cause outpatient hospital visits compared with $40.1 \%$ of patients without cognitive dysfunction; however, the difference between cohorts was no longer statistically significant $(P=0.108)$. Over 6 months, more patients with cognitive dysfunction had an all-cause emergency room visit compared with patients without cognitive dysfunction $(29.0 \%$ vs. $19.5 \%, P=0.009)$. Over that span, significantly more patients with cognitive dysfunction than without had an outpatient visit for depressive disorder $(8.3 \%$ vs. $4.2 \%, P=0.044)$.

\section{Follow-up All-Cause Costs}

At 3 months, the difference in unadjusted all-cause total costs was not statistically significant $(P=0.113$; Tables 2 and 4$)$. By 6 months, however, the unadjusted all-cause total costs were significantly higher $(\$ 6,707)$ for patients with cognitive dysfunction compared with those without cognitive dysfunction $(\$ 3,938, P=0.040$; Tables 2 and 4). For both follow-up periods, the largest cost drivers were office and outpatient visits, which accounted for half of total costs, followed by inpatient costs, which accounted for one-quarter of total costs. The difference between the 2 cohorts was significantly higher $(P=0.012$ and $0.011)$ for outpatient hospital visits in the cognitive dysfunction cohort for the 3- and 6-month periods and emergency room visits $(P=0.047)$ for the 6 -month period. Median costs were similar across the 2 cohorts for the 3-month and 6-month total costs and cost components (i.e., office, outpatient hospital, emergency room, inpatient, other medical, and pharmacy).

The adjusted gamma model yielded significant differences in mean all-cause costs (Table 2). At 3 months, patients with cognitive dysfunction had higher adjusted all-cause costs compared with patients in the cognitive normal cohort ( $\$ 3,309$ vs. $\$ 2,157$, $P=0.002$ ). Patients with cognitive dysfunction also had higher 6-month adjusted all-cause costs than those without cognitive dysfunction ( $\$ 4,793$ vs. $\$ 3,683, P=0.034)$. Gamma models yielded cost ratios (see Appendix F, available in online article) for all-cause costs at 1.53 times higher (95\% confidence interval $[C I]=1.17-2.02, P=0.002)$ for patients with cognitive dysfunction than for patients without cognitive dysfunction at 3 months. In addition, baseline Quan-Charlson score $>0$ and full-time employment were significantly and positively associated with all-cause costs during the 3-month follow-up period. The cost ratio at 6 months for all-cause costs was 1.30 times higher (95\% $\mathrm{CI}=1.02-1.66, P=0.034)$ for the cognitive dysfunction cohort than the cognitive normal cohort. Baseline Quan-Charlson score $>0$ was significantly and positively associated with all-cause costs during the 6-month follow-up period. 


\section{Association Between Cognitive Function and Health Care Costs 3 Months and 6 Months After Initiating Antidepressant Medication for Depressive Disorders}

\section{TABLE 3 All-Cause and Depressive Disorder-Related Health Care Utilization}

over 3 Months and 6 Months by Cohort

\begin{tabular}{|c|c|c|c|c|c|c|}
\hline \multirow{3}{*}{3 Months } & \multicolumn{2}{|c|}{$\begin{array}{l}\text { All-Cause } \\
\text { Utilization }\end{array}$} & \multirow{3}{*}{$\frac{P \text { Value }}{0.567}$} & \multicolumn{2}{|c|}{$\begin{array}{c}\text { Depressive Disorder-Related } \\
\text { Utilization }\end{array}$} & \multirow{3}{*}{$\frac{P \text { Value }}{0.866}$} \\
\hline & $\begin{array}{l}\text { Cognitive } \\
\text { Normal } \\
(\mathbf{n}=308)\end{array}$ & $\begin{array}{l}\text { Cognitive } \\
\text { Dysfunction } \\
(\mathbf{n}=255)\end{array}$ & & $\begin{array}{l}\text { Cognitive } \\
\text { Normal } \\
(\mathrm{n}=308)\end{array}$ & $\begin{array}{l}\text { Cognitive } \\
\text { Dysfunction } \\
(\mathbf{n}=255)\end{array}$ & \\
\hline & $273(88.6)$ & $222(87.1)$ & & $97 \quad(31.5)$ & $82(32.2)$ & \\
\hline Office visit & $271(88.0)$ & $219 \quad(85.9)$ & 0.459 & $94 \quad(30.5)$ & $79 \quad(31.0)$ & 0.906 \\
\hline Outpatient hospital visit & $76 \quad(24.7)$ & $83(32.6)$ & 0.039 & $11 \quad(3.6)$ & $12 \quad(4.7)$ & 0.498 \\
\hline ER visit, n (\%) & 42 (13.6) & $45 \quad(17.7)$ & 0.190 & $(1.0)$ & $(3.1)$ & 0.065 \\
\hline Inpatient hospital stay, n (\%) & $12 \quad(3.9)$ & $\begin{array}{ll}6 & (2.4) \\
\end{array}$ & 0.300 & $6 \quad(2.0)$ & $3 \quad(1.2)$ & 0.467 \\
\hline Prescriptions, $\mathrm{n}(\%)$ & $306 \quad(99.4)$ & $251 \quad(98.4)$ & 0.290 & $305(99.0)$ & $250(98.0)$ & 0.325 \\
\hline Ambulatory visit, mean (SD) & $4.47 \quad(4.7)$ & $5.05 \quad(5.7)$ & 0.198 & $0.73 \quad(1.7)$ & $0.71 \quad(1.4)$ & 0.892 \\
\hline Office visit & $3.62 \quad(3.7)$ & $3.80 \quad(4.2)$ & 0.596 & $0.66 \quad(1.5)$ & $(1.3)$ & 0.947 \\
\hline Outpatient hospital visit & $(2.1)$ & $1.25 \quad(2.7)$ & 0.068 & $(0.4)$ & 0.06 & 0.756 \\
\hline ER visit, mean (SD) & $(0.8)$ & 0.36 & 0.191 & $(0.2)$ & 0.07 & 0.063 \\
\hline Inpatient hospital stay, mean (SD) & $(0.2)$ & 0.04 & 0.990 & $(0.1)$ & $(0.2)$ & 0.758 \\
\hline Length of hospital stay, days, mean (SD) & 0.22 & (3.3) & 0.536 & $(1.6)$ & $(2.2)$ & 0.830 \\
\hline Prescriptions, mean (SD) & $(5.0)$ & $(5.8)$ & 0.096 & $3.17 \quad(1.8)$ & $(2.2)$ & 0.352 \\
\hline \multirow[b]{2}{*}{6 Months } & \multicolumn{2}{|c|}{$\begin{array}{l}\text { All-Cause } \\
\text { Utilization }\end{array}$} & & \multicolumn{2}{|c|}{$\begin{array}{c}\text { Depressive Disorder-Related } \\
\text { Utilization }\end{array}$} & \multirow[b]{2}{*}{$P$ Value } \\
\hline & $\begin{array}{l}\text { Cognitive } \\
\text { Normal } \\
(\mathbf{n}=307)\end{array}$ & $\begin{array}{c}\text { Cognitive } \\
\text { Dysfunction } \\
(\mathbf{n}=252)\end{array}$ & $P$ Value & $\begin{array}{c}\text { Cognitive } \\
\text { Normal } \\
(\mathrm{n}=307)\end{array}$ & $\begin{array}{l}\text { Cognitive } \\
\text { Dysfunction } \\
(\mathbf{n}=252)\end{array}$ & \\
\hline Ambulatory visit, $\mathrm{n}(\%)$ & $290 \quad(94.5)$ & $238 \quad(94.4)$ & 0.993 & $117(38.1)$ & $96 \quad(38.1)$ & 0.997 \\
\hline Office visit & $289(94.1)$ & $235(93.3)$ & 0.668 & $114(37.1)$ & $88 \quad(35.9)$ & 0.588 \\
\hline Outpatient hospital visit & $123(40.1)$ & $118 \quad(46.8)$ & 0.108 & $13 \quad(4.2)$ & $21 \quad(8.3)$ & 0.044 \\
\hline ER visit, n (\%) & $60 \quad(19.5)$ & $73(29.0)$ & 0.009 & $(1.0)$ & $(2.8)$ & 0.110 \\
\hline Inpatient hospital stay, n (\%) & $17 \quad(5.5)$ & $15 \quad(5.6)$ & 0.993 & $8 \quad(2.6)$ & $5 \quad(2.0)$ & 0.627 \\
\hline Prescriptions, $\mathrm{n}(\%)$ & $305(99.4)$ & $249(98.8)$ & 0.501 & $304(99.0)$ & $249(98.8)$ & 0.808 \\
\hline Ambulatory visit, mean (SD) & $7.64 \quad(8.1)$ & $8.75(10.6)$ & 0.174 & $1.03 \quad(2.4)$ & $1.02 \quad(2.2)$ & 0.958 \\
\hline Office visit & $(6.4)$ & $6.43 \quad(8.2)$ & 0.538 & $(2.2)$ & $(2.1)$ & 0.882 \\
\hline Outpatient hospital visit & $(3.5)$ & 2.34 & 0.050 & $(0.1)$ & $(0.3)$ & 0.605 \\
\hline ER visit, mean (SD) & $(1.4)$ & $(1.8)$ & 0.259 & $(0.2)$ & 0.09 & 0.154 \\
\hline Inpatient hospital stay, mean (SD) & $(0.3)$ & 0.08 & 0.507 & $(0.2)$ & 0.02 & 0.875 \\
\hline Length of hospital stay, days, mean (SD) & 0.29 & $0.50 \quad(3.3)$ & 0.365 & $(1.6)$ & 0.23 & 0.658 \\
\hline Prescriptions, mean (SD) & 12.34 & $13.83(11.0)$ & 0.090 & $(3.4)$ & $(3.7)$ & 0.721 \\
\hline
\end{tabular}

\section{Follow-up Depressive Disorder-Related Costs}

The difference in unadjusted depressive disorder-related total costs were not statistically significant by 3 months $(P=0.536$; Tables 2 and 4$)$ or by 6 months $(P=0.387)$. The largest cost drivers were inpatient care, which accounted for approximately two-thirds of the total depressive disorder-related costs, followed by outpatient hospital visits, office visits, and pharmacy costs, with each accounting for less than onequarter of the costs. The outpatient hospital costs at 6 -months post-index were significantly higher $(P=0.030)$ among the cognitive dysfunction cohort compared with the cognitive normal cohort. The median costs were similar across the 2 cohorts for the 3-month and 6-month total costs and cost components.
In the adjusted gamma model, patients with cognitive dysfunction had significantly higher 3-month depressive disorderrelated total costs (\$718) compared with patients without cognitive dysfunction ( $\$ 406, P<0.001$; Table 2$)$. The cost ratio (see Appendix $F$, available in online article) indicated 1.77 times (95\% CI $=1.30-2.41, P<0.001$ ) higher depressive disorder-related costs for patients with cognitive dysfunction than those without.

In addition, baseline Quan-Charlson score $>0$ and fulltime employment were significantly and positively associated with all-cause costs during the 3-month follow-up period. At 6 months, the ratio was 1.30 times (95\% CI=0.98-1.72, $P=0.071$ ) higher costs for patients with cognitive dysfunction. Baseline Quan-Charlson score $>0$ and full-time employment 


\begin{tabular}{|c|c|c|c|c|c|c|c|}
\hline \multirow{2}{*}{\multicolumn{2}{|c|}{3 Months }} & \multicolumn{2}{|c|}{ All-Cause Costs } & \multirow{3}{*}{$\frac{P \text { Value }}{0.113}$} & \multicolumn{2}{|c|}{ Depressive Disorder-Related Costs } & \multirow{3}{*}{$\frac{P \text { Value }}{0.536}$} \\
\hline & & $\begin{array}{c}\text { Cognitive } \\
\text { Normal } \\
(\mathbf{n}=308, \$)\end{array}$ & $\begin{array}{c}\text { Cognitive } \\
\text { Dysfunction } \\
(\mathrm{n}=255, \$)\end{array}$ & & $\begin{array}{c}\text { Cognitive } \\
\text { Normal } \\
(\mathrm{n}=308, \$)\end{array}$ & $\begin{array}{l}\text { Cognitive } \\
\text { Dysfunction } \\
(\mathbf{n}=255, \$)\end{array}$ & \\
\hline \multirow[t]{2}{*}{ Total } & mean (SD) & $2,312 \quad(8,266)$ & $3,948(14,643)$ & & $564 \quad(4,099)$ & $951 \quad(9,255)$ & \\
\hline & median & 669 & 695 & & 99 & 91 & \\
\hline \multirow[t]{2}{*}{ Office visit } & mean $(\mathrm{SD})$ & $(1,441)$ & $825 \quad(4,576)$ & 0.317 & $(142)$ & $(132)$ & 0.797 \\
\hline & median & 276 & 247 & & 0 & 0 & \\
\hline \multirow{2}{*}{$\begin{array}{l}\text { Outpatient } \\
\text { hospital visit }\end{array}$} & mean (SD) & $(1,212)$ & $1,139 \quad(4,567)$ & 0.012 & $(320)$ & $(427)$ & 0.530 \\
\hline & median & 0 & 0 & & 0 & 0 & \\
\hline \multirow[t]{2}{*}{ Emergency room } & mean $(\mathrm{SD})$ & $(149)$ & $(222)$ & 0.078 & $(45)$ & $(38)$ & 0.516 \\
\hline & median & 0 & 0 & & 0 & 0 & \\
\hline \multirow[t]{2}{*}{ Inpatient } & mean (SD) & $(7,677)$ & $1,238(11,550)$ & 0.676 & $337 \quad(4,063)$ & $674 \quad(9,186)$ & 0.587 \\
\hline & median & 0 & 0 & & 0 & 0 & \\
\hline \multirow[t]{2}{*}{ Other medical } & mean (SD) & $(484)$ & $(873)$ & 0.308 & $(50)$ & $(427)$ & 0.309 \\
\hline & median & 0 & 0 & & 0 & 0 & \\
\hline \multirow[t]{2}{*}{ Pharmacy } & mean $(\mathrm{SD})$ & $(1,122)$ & $508 \quad(1,151)$ & 0.114 & $(206)$ & $(215)$ & 0.878 \\
\hline & median & 157 & 163 & & 50 & 40 & \\
\hline \multirow{2}{*}{\multicolumn{2}{|c|}{6 Months }} & \multicolumn{2}{|c|}{ All-Cause Costs } & & \multicolumn{2}{|c|}{ Depressive Disorder-Related Costs } & \\
\hline & & $\begin{array}{c}\text { Cognitive } \\
\text { Normal } \\
(\mathbf{n}=307, \$)\end{array}$ & $\begin{array}{c}\text { Cognitive } \\
\text { Dysfunction } \\
(\mathrm{n}=252, \$)\end{array}$ & $P$ Value & $\begin{array}{c}\text { Cognitive } \\
\text { Normal } \\
(\mathbf{n}=307)\end{array}$ & $\begin{array}{l}\text { Cognitive } \\
\text { Dysfunction } \\
(\mathbf{n}=252)\end{array}$ & $P$ Value \\
\hline \multirow[t]{2}{*}{ Total } & mean (SD) & $3,938(10,488)$ & $6,707(19,038)$ & 0.040 & $759 \quad(4,282)$ & $1,316 \quad(9,446)$ & 0.387 \\
\hline & median & 1,252 & 1,382 & & 150 & 147 & \\
\hline \multirow[t]{2}{*}{ Office visit } & mean (SD) & $(1,956)$ & $1,516 \quad(9,023)$ & 0.259 & $(205)$ & $(204)$ & 0.674 \\
\hline & median & 459 & 399 & & 0 & 0 & \\
\hline \multirow{2}{*}{$\begin{array}{l}\text { Outpatient hospital } \\
\text { visit }\end{array}$} & mean (SD) & $(2,619)$ & $(7,313)$ & 0.011 & (343) & $(1,090)$ & 0.030 \\
\hline & median & 0 & 0 & & 0 & 0 & \\
\hline \multirow[t]{2}{*}{ Emergency room } & mean (SD) & $(212)$ & $(674)$ & 0.047 & (46) & $(55)$ & 0.545 \\
\hline & median & 0 & 0 & & 0 & 0 & \\
\hline \multirow[t]{2}{*}{ Inpatient } & mean (SD) & $(8,162)$ & $1,759(12,090)$ & 0.544 & $423 \quad(4,228)$ & $(9,340)$ & 0.548 \\
\hline & median & 0 & 0 & & 0 & 0 & \\
\hline \multirow[t]{2}{*}{ Other medical } & mean (SD) & $(699)$ & $262 \quad(1,006)$ & 0.267 & $(51)$ & $(433)$ & 0.263 \\
\hline & median & 2 & 8 & & 0 & 0 & \\
\hline Pharmacy & mean (SD) & $687 \quad(2,346)$ & $844 \quad(1,649)$ & 0.353 & $(388)$ & $(325)$ & 0.471 \\
\hline & median & 271 & 283 & & 82 & 68 & \\
\hline
\end{tabular}

were significantly and positively associated with all-cause costs during the 6-month follow-up period.

\section{Discussion}

In this study, we classified patients with depressive disorder from an ambulatory care setting into cognitive dysfunction or cognitive normal cohorts, based on standard assessments of cognitive function. We compared all-cause and depressive disorder-related costs across the 2 cohorts to determine if costs for patients with cognitive dysfunction were higher than costs for patients without cognitive dysfunction. In addition, the types of health care services that accounted for the costs for each cohort were identified. For all patients, regardless of cognitive dysfunction, the main drivers of all-cause costs prior to and following antidepressant initiation were office visits, outpatient hospital visits, and inpatient costs. The main driver of depressive disorder-related costs was inpatient costs. These results for depressive disorder-related costs are consistent with results from a systematic review of cost-of-illness studies in depression that found inpatient costs to be cost drivers, followed by outpatient costs and pharmacy costs. ${ }^{5}$

The cognitive normal cohort had more patients who were white and employed full time; this cohort also had higher education and fewer comorbidities. Thus, the associations between cognitive dysfunction and total follow-up costs were tested with adjustment for potential confounders. After 
adjustment, compared with patients without cognitive dysfunction, patients with cognitive dysfunction had significantly higher all-cause and depressive disorder-related health care costs over 3 months and higher all-cause costs over 6 months. Over 6 months, the difference in unadjusted all-cause costs between patients with cognitive dysfunction and those without was $\$ 2,769$ ( $\$ 6,707-\$ 3,938)$; controlling for potential confounders, the difference was $\$ 1,110(\$ 4,793-\$ 3,683)$. These differences in all-cause costs may be attributed to the greater need for follow-up services in patients with cognitive dysfunction, that is, to the additional cost of treating cognitive dysfunction following antidepressant treatment. Likewise, the difference over 6 months in unadjusted depressive disorder-related costs was $\$ 557$ ( $\$ 1,316-\$ 759)$; controlling for confounders, it was $\$ 177$ (\$771-\$594). These differences in costs could also be attributable to the additional depressive disorder-related treatment of cognitive dysfunction (greater need for follow-up services in patients with cognitive dysfunction).

We are not aware of any published work measuring the additional costs of treating cognitive dysfunction in patients with depressive disorders against which to compare our results. However, 2 studies did measure the additional costs of other concomitant conditions in patients with MDD. One study, a U.S. claims-based study, found increased costs in patients with MDD who were taking insomnia medication..$^{25}$ Patients taking insomnia medication had higher annual adjusted all-cause costs ( $\$ 3,918$ more) and adjusted MDD-related costs ( $\$ 537$ more), compared with MDD patients not taking insomnia medication. Another U.S. claims-based study found that patients with MDD and anxiety had higher annual costs $(\$ 10,031)$ than patients with MDD only $(\$ 6,681)$, a difference of $\$ 3,350 .{ }^{26}$ Our unadjusted all-cause cost of treating patients with cognitive dysfunction over 6 months was $\$ 2,769$, and our adjusted cost was $\$ 1,110$; annualized, these are $\$ 5,538$ and $\$ 2,220$. These numbers are comparable with the all-cause costs of insomnia at $\$ 3,918$ and anxiety at $\$ 3,350$. Our unadjusted depressive disorder-related cost of treating patients with cognitive dysfunction over 6 months was $\$ 557$, and our adjusted cost was $\$ 177$, which when annualized are $\$ 1,114$ and $\$ 354$ - comparable to the MDD-related cost of insomnia at $\$ 537$.

The annual cost to treat patients with MDD only $(\$ 6,681)$ in the study of MDD with anxiety, ${ }^{26}$ and the unadjusted cost to treat MDD patients without insomnia $(\$ 7,015),{ }^{25}$ can be compared with the cost of our depressive-disorder patients without cognitive dysfunction to compare the costs of treating depression. Our annualized (unadjusted all-cause $\$ 7,876[\$ 3,938 \times 2]$ and adjusted all-cause $\$ 7,366[\$ 3,683 \times 2]$ ) costs were similar. The mean unadjusted annual MDD-related cost in patients without insomnia was $\$ 824,{ }^{25}$ compared with the depressive disorder-related costs of our patients without cognitive dysfunction, when our costs are annualized (unadjusted depressive disorder-related $\$ 1,518[\$ 759 \times 2]$ and adjusted depressive disorder-related $\$ 1,188[\$ 594 \times 2]$ ). The systematic review of cost-of-illness studies of depression found an average annual direct medical cost of $\$ 1,000$ to $\$ 2,500$.

Another study found that patients with diabetes and a dementia diagnosis in 2005 had total average Medicare payments in 2006 of $\$ 20,655$ compared with $\$ 12,979$ in patients with diabetes and no dementia diagnosis, for a difference of $\$ 7,676 .{ }^{11}$ This cost of dementia is higher than our annualized unadjusted all-cause cost of $\$ 5,538$ and our annualized adjusted all-cause cost of $\$ 2,220$ for treating cognitive dysfunction. Our lower costs for cognitive dysfunction is not surprising because patients with dementia have more severe cognitive dysfunction, and there are other additional confounders, such as comorbidities, age, and medication. Although we did not measure the long-term consequences of not treating cognitive dysfunction, our data support the consideration that cognitive dysfunction is an important symptom with cost implications similar to other chronic illnesses.

In fact, a recent meta-analysis identified cognitive deficits in patients with first-episode MDD and suggested that cognitive deficits appear to be a feasible early marker and target for early intervention in MDD. ${ }^{19}$ Patients in our study with cognitive dysfunction had higher all-cause and depressive disorder-related costs compared with patients with no cognitive dysfunction, and this difference persisted for all-cause costs through 6 months. Therefore, identifying and treating cognitive dysfunction and depressive disorder may save downstream costs to the health care system.

\section{Limitations}

We acknowledge several limitations of this study. The original study was designed to compare clinical outcomes between groups, and the secondary objective was to compare costs. ${ }^{15}$ Direct medical costs were included in the results; however, indirect costs can be substantial and were not included. ${ }^{5}$ The total follow-up costs were adjusted for potential confounders; however, the component follow-up costs (and baseline costs) were not adjusted because several components of total followup costs had zero costs, which is not conducive to a modelbased adjustment. The severity of baseline depression could not be determined and therefore was also not included as a potential confounder. This sample was drawn from a much larger sample population, and a selection bias could exist. However, this lack of representativeness does not invalidate the results of the study, since the mechanisms underlying higher health care costs for depressed patients with cognitive dysfunction can be considered as independent of the representativeness of the sample, as with most relations identified in epidemiological studies. ${ }^{27}$ The sample includes only patients with commercial insurance, who were aged 18-65 years and 80\% female, and most of whom were employed, so it may not be representative of the general population. The nature of claims data collection limited the speed with which identification, recruitment, and participation in the survey followed the index date 


\section{Association Between Cognitive Function and Health Care Costs 3 Months and 6 Months After Initiating Antidepressant Medication for Depressive Disorders}

(antidepressant initiation). Patients with cognitive dysfunction at the index date may have benefitted from treatment and been classified as cognitive normal by the time they received their telephone interviews. This may bias the results to having a higher cost in the cognitive normal group because of earlier cognitive dysfunction. The remaining patients with cognitive dysfunction may have had a higher severity of cognitive dysfunction and raised the cost in the cognitive dysfunction group. Another limitation was having cognitive dysfunction measured at 1 time point only. By 6 months, the difference between groups in depressive disorder-related costs was no longer significant after adjusting for confounders, and it could be that patients may not have remained in the original classification of cognitive normal or cognitive dysfunction. Our standard assessments were provided over the telephone. Although the interviewers were trained specifically for this study by neuropsychologists, they were not neuropsychologists themselves. In addition, our method of collectively taking results of 4 cognitive assessment measures to classify patients by cognitive function has not been validated, although each of these cognitive instruments has been validated individually.

\section{Conclusions}

In this study of patients with depressive disorder, all-cause and depressive disorder-related adjusted health care costs over 3 months were significantly higher in patients with cognitive dysfunction compared with those without cognitive dysfunction. By 6 months, although the difference in depressive disorder-related costs was no longer significant after adjusting for confounders, the adjusted all-cause costs remained significantly higher for patients with cognitive dysfunction. These data support the idea that cognitive dysfunction is associated with higher health care costs in depressed individuals and may be a useful target symptom for future treatment advances.

\section{Authors}

VALERY WALKER, MSc, is Director, Health Economics and Outcomes Research; JONATHAN L. KURLANDER, MS, is Director, Health Economics and Outcomes Research; and BREANNA ESSOI, $\mathrm{MPH}$, is Project Manager, Health Economics and Outcomes Research, Optum, Eden Prairie, Minnesota. HARIDARSHAN PATEL, PharmD, is a Fellow, and JENNIFER C. SAMP, PharmD, MS, is a former Fellow, Takeda Development Center Americas, Deerfield, Illinois. JIAO YANG, PhD, is Principal Statistician, and ATUL R. MAHABLESHWARKAR, MD, is Senior Medical Director, Takeda Development Center Americas, Deerfield, Illinois. KASEM S. AKHRAS, PharmD, is Head, Market Access-Middle East and North Africa, Novartis Pharma Services, Dubai, United Arab Emirates.

AUTHOR CORRESPONDENCE: Valery Walker, MSc, Director, Health Economic and Outcomes Research, Optum, 12125 Technology Dr., Eden Prairie, MN 55344. Tel.: 289.313.6030; Fax: 289.313.6043; E-mail:valery.walker@optum.com.

\section{DISCLOSURES}

This study was funded by Takeda Pharmaceutical Company, Ltd., and H. Lundbeck A/S. Walker, Essoi, and Kurlander were paid consultants for the project. Akhras was an employee of Takeda Pharmaceutical Company, Ltd., at the time of the study. Samp was supported by a fellowship funded by Takeda Pharmaceutical Company, Ltd., at the time of the study. Patel is supported by a fellowship funded by Takeda Pharmaceutical Company, Ltd. Mahableshwarkar and Yang are current employees of Takeda Pharmaceutical Company, Ltd.

Study concept and design were contributed by Walker, Samp, and Akhras, with assistance from Kurlander, Essoi, and Mahableshwarkar. Data were collected by Essoi, Walker, and Kurlander and interpreted by Patel, Yang, Mahableshwarkar, and Kurlander, with assistance from Walker. The manuscript was written by primarily by Walker and Kurlander, with assistance from the other authors, and revised by Walker, Patel, and Kurlander, with assistance from the other authors.

\section{ACKNOWLEDGMENTS}

The authors would like to thank Caroline Jennermann, who provided medical writing support and was funded by Takeda Pharmaceutical Company, Ltd. The authors would like to thank Gwenyth Van Trieste and Rebecca Banua and the employees at HumRRO and Braun Research, Inc., who were responsible for conducting the telephone interviews. The authors thank Paul Maruff, Judith Jaeger, and Lisle Kingery from Cogstate for their work during protocol development and analysis, and Vanitha Krishna, Vicky Lo, and Athena Nomikos (from Cogstate), and J. Jason van Steenburg and Steve Michael (consultants at Cogstate) for their work in interviewer training. The authors would also like to thank the following individuals at Optum who were involved in the study at various stages: Francisca Azocar, Martha Bayliss, Susan Brenneman, Paul Buzinec, Christa Castaneda, Randy Gerdes, Yvette Greiner, Margaret Good, Nancy Hartman, Alyssa Goolsby Hunter, Carolyn Martin, Jeffrey McPheeters, Angela Watkins, and Bryan Zachau.

\section{REFERENCES}

1. Murray CJ, Lopez AD. Evidence-based health policy-lessons from the Global Burden of Disease Study. Science. 1996;274(5288):740-43.

2. Kessler RC, Berglund P, Demler O, et al. The epidemiology of major depressive disorder: results from the National Comorbidity Survey Replication (NCS-R). JAMA. 2003;289(23):3095-105

3. National Comorbidity Survey Replication. Table 1. Lifetime prevalence of DSM-IV/WMH-CIDI disorders by sex and cohort. 2007. Available at: http://www.hcp.med.harvard.edu/ncs/ftpdir/NCS-R_Lifetime_Prevalence_ Estimates.pdf. Accessed July 17, 2015

4. Greenberg PE, Kessler RC, Birnbaum HG, et al. The economic burden of depression in the United States: how did it change between 1990 and 2000? J Clin Psychiatry. 2003;64(12):1465-75.

5. Luppa M, Heinrich S, Angermeyer MC, König HH, Riedel-Heller SG. Cost-of-illness studies of depression: a systematic review. J Affect Disord. 2007;98(1-2):29-43

6. Casacalenda N, Perry JC, Looper K. Remission in major depressive disorder: a comparison of pharmacotherapy, psychotherapy, and control conditions. Am J Psychiatry. 2002;159(8):1354-60.

7. American Psychiatric Association. Depressive disorders. In: Diagnostic and Statistical Manual of Mental Disorders, 5th ed., Arlington, VA: American Psychiatric Publishing; 2013

8. Paelecke-Habermann Y, Pohl J, Leplow B. Attention and executive functions in remitted major depression patients. J Affect Disord. 2005;89(1-3):125-35.

9. Weiland-Fiedler P, Erickson K, Waldeck T, et al. Evidence for continuing neuropsychological impairments in depression. J Affect Disord. 2004;82(2):253-58. 
10. Airaksinen E, Wahlin A, Larsson M, Forsell Y. Cognitive and social functioning in recovery from depression: results from a population-based threeyear follow-up. J Affect Disord. 2006;96(1-2):107-10.

11. Bynum J. Characteristics, costs $\&$ health service use for Medicare beneficiaries with a dementia diagnosis. Alzheimer's Association. January 2009. Available at: http://collectiveactionlab.com/sites/default/files/BynumCharacteristics\%20Costs\%20and\%20Health\%20Service\%20Use\%20Reports. pdf. Accessed July 17, 2015.

12. U.S. Census Bureau. Census regions and divisions of the United States. 2011. Available at: http://www2.census.gov/geo/pdfs/maps-data/maps/reference/us_regdiv.pdf. Accessed July 17, 2015.

13. Healthcare Cost and Utilization Project (HCUP). Comorbidity software, version 3.7. 2013. Available at: http://www.hcup-us.ahrq.gov/toolssoftware/ comorbidity/comorbidity.jsp. Accessed July 17, 2015.

14. Centers for Disease Control and Prevention. International Classification of Diseases, Ninth Revision, Clinical Modification (ICD-9-CM). Page updated June 2013. Available at: http://www.cdc.gov/nchs/icd/icd $9 \mathrm{~cm} . h t m$. Accessed July 17, 2015.

15. Samp JC, Erlander JL, Walker V, Essoi B, Patel H, Akhras KS. Assessment of functional outcomes in individuals treated for depression in the United States based on a cognitive dysfunction classification. Poster presented at: U.S. Psychiatric \& Mental Health Congress; September 30-October 2, 2013; Las Vegas, NV.

16. Benedict RHB, Schretlen D, Groninger L, Brandt J. Hopkins Verbal Learning Test-Revised: normative data and analysis of inter-form and testretest reliability. Clin Neuropsychol. 1998;12(1):43-55.

17. Wechsler D. Wechsler Adult Intelligence Scale-Third Edition (WMS-III) Administration and Scoring Nanual. San Antonio, TX: The Psychological Corporation; 1997.
18. Delis D, Kaplan EB, Kramer J. Delis-Kaplan Executive Function System (D-KEFS). San Antonio, TX: The Psychological Corporation; 2001.

19. Lee RS, Hermens DF, Porter MA, Redoblado-Hodge MA. A meta-analysis of cognitive deficits in first-episode major depressive disorder. J Affect Disord. 2012;140(2):113-24

20. Veiel HO. A preliminary profile of neuropsychological deficits associated with major depression. J Clin Exp Neuropsychol. 1997;19(4):587-603.

21. Snyder HR. Major depressive disorder is associated with broad impairments on neuropsychological measures of executive function: a meta-analysis and review. Psychol Bull. 2013;139(1):81-132.

22. Ingraham LJ, Aiken CB. An empirical approach to determining criteria for abnormality in test batteries with multiple measures. Neuropsychology. 1996;10(1):120-24.

23. Manning WG, Mullahy J. Estimating log models: to transform or not to transform? J Health Econ. 2001;20(4):461-94.

24. Manning WG. The logged dependent variable, heteroscedasticity, and the retransformation problem. J Health Econ. 1998;17(3):283-95.

25. Tian H, Abouzaid S, Gabriel S, Kahler KH, Kim E. Resource utilization and costs associated with insomnia treatment in patients with major depressive disorder. Prim Care Companion CNS Disord. 2012;14(5).

26. François C, Despiégel N, Maman K, Saragoussi D, Auquier P. Anxiety disorders, major depressive disorder and the dynamic relationship between these conditions: treatment patterns and cost analysis. J Med Econ. 2010;13(1):99-109.

27. Rothman KJ, Gallacher JE, Hatch EE. Why representativeness should be avoided. Int J Epidemiol. 2013;42(4):1012-14. 


\section{Association Between Cognitive Function and Health Care Costs 3 Months and 6 Months After Initiating Antidepressant Medication for Depressive Disorders}

\section{APPENDIX A Inclusion and Exclusion Criteria}

Inclusion Criteria

At least 1 pharmacy claim for an antidepressant listed in Appendix B during the identification period based on the most currently available pharmacy claims data (approximately August and September 2012). Identification of the antidepressant will occur at the end of the month when the claims database has been updated. The identification period will include the current month (up to the date the database has been updated) and the prior month. The index date will be defined as the date of the first antidepressant pharmacy claim for the antidepressant. The antidepressant a patient receives on the index date will be defined as the index antidepressant.

No pharmacy claim for any antidepressant medication 6 months prior to initiation of index antidepressant; the 6-month period before the index date will be defined as the baseline period.

Aged 18 to 65 years as of the year of the index date.

Self-reported major depressive disorder diagnosis in the invitation reply and patient survey.

Self-reported confirmation of no diagnoses of schizophrenia, bipolar disorder, epilepsy, multiple sclerosis, Parkinson's disease, Alzheimer's disease, or Huntington's disease in the invitation reply and patient survey.

Continuous enrollment in a large commercial U.S. health plan affiliated with OptumInsight during the baseline period.

Mental health coverage with OptumHealth Behavioral Solutions (OHBS).

Willingness to sign and return the informed consent and health information release forms

Willingness and ability to participate in a telephone interview conducted in English.

\section{Exclusion Criteria}

Expiration of health plan enrollment 6 months or less from the index date. Any claim with a primary or secondary diagnosis code for schizophrenia, bipolar disorder, Alzheimer's disease, epilepsy disorder, Huntington's disease, Parkinson's disease, and multiple sclerosis during the 1 year prior to initiation of index antidepressant.

Any prescription for 1 of the following conditions: schizophrenia, bipolar disorder, Alzheimer's disease, epilepsy disorder, Huntington's disease, Parkinson's disease, and multiple sclerosis during the baseline period.

\section{APPENDIX B List of Antidepressants}

Drug Class

Aminoketone (DNRI)

Generic Name (Brand Name)

\begin{tabular}{|c|c|}
\hline & \multirow[b]{2}{*}{ bupropion XL (Budeprion XL) } \\
\hline & \\
\hline & bupropion (Wellbutrin) \\
\hline & bupropion SR (Wellbutrin SR) \\
\hline & bupropion XL (Wellbutrin XL) \\
\hline & bupropion XL (Forfivo XL) \\
\hline & buproprion (Aplenzin) \\
\hline & bupropion/diet suppl (Appbutamone) \\
\hline \multirow[t]{12}{*}{ SSRI } & citalopram (Celexa) \\
\hline & escitalopram (Lexapro) \\
\hline & fluoxetine (Prozac) \\
\hline & fluoxetine (Rapiflux) \\
\hline & fluoxetine (Sarafem) \\
\hline & fluoxetine (Selfemra) \\
\hline & fluoxetine/diet suppl (Sentroxatin) \\
\hline & fluoxetine/diet suppl (Gaboxetine) \\
\hline & paroxetine (Paxil) \\
\hline & paroxetine (Paxil CR) \\
\hline & paroxetine (Pexeva) \\
\hline & sertraline (Zoloft) \\
\hline \multirow[t]{4}{*}{ SNRI } & desvenlafaxine (Pristiq) \\
\hline & duloxetine (Cymbalta) \\
\hline & venlafaxine (Effexor) \\
\hline & venlafaxine (Effexor XR) \\
\hline \multirow[t]{4}{*}{ Other } & mirtazapine (Remeron) \\
\hline & nefazodone (Serzone) \\
\hline & trazodone (Desyrel) \\
\hline & vilazodone hcl (Viibryd) \\
\hline
\end{tabular}

$C R=$ controlled release DNRI = dopamine-norepinephrine reuptake inhibitor; $S N R I=$ serotonin and norepinephrine reuptake inhibitor; $S R=$ sustained release; $S S R I=$ selective serotonin reuptake inhibitor; suppl=supplement; $X L=$ extended release; $X R=$ extended release. 


\section{Association Between Cognitive Function and Health Care Costs 3 Months and 6 Months After Initiating Antidepressant Medication for Depressive Disorders}

\begin{tabular}{|c|c|c|c|}
\hline \multicolumn{4}{|c|}{ APPENDIX C ICD-9-CM Diagnosis Codes for Patient Exclusion } \\
\hline Code & Description & \multicolumn{2}{|r|}{ Description } \\
\hline 331.0 & Alzheimer's disease & 295.73 & Schizoaffective disorder, subchronic with acute exacerbation \\
\hline 331.82 & Dementia with parkinsonism & 295.74 & Schizoaffective disorder, chronic with acute exacerbation \\
\hline 332.0 & Paralysis agitans & 295.75 & Schizoaffective disorder, in remission \\
\hline 333.4 & Huntington's chorea & 295.80 & Other specified types of schizophrenia, unspecified condition \\
\hline 340 & Multiple sclerosis & 295.81 & Other specified types of schizophrenia, subchronic condition \\
\hline 295.00 & Simple schizophrenia, unspecified condition & 295.82 & Other specified types of schizophrenia, chronic condition \\
\hline 295.01 & Simple schizophrenia, subchronic condition & \multirow[t]{2}{*}{295.83} & \multirow{2}{*}{$\begin{array}{l}\text { Other specified types of schizophrenia, subchronic condition with } \\
\text { acute exacerbation }\end{array}$} \\
\hline 295.02 & Simple schizophrenia, chronic condition & & \\
\hline 295.03 & Simple schizophrenia, subchronic condition with acute exacerbation & \multirow[t]{2}{*}{295.84} & \multirow{2}{*}{$\begin{array}{l}\text { Other specified types of schizophrenia, chronic condition with } \\
\text { acute exacerbation }\end{array}$} \\
\hline 295.04 & Simple schizophrenia, chronic condition with acute exacerbation & & \\
\hline 295.05 & Simple schizophrenia, in remission & \multirow{2}{*}{$-\frac{295.85}{295.90}$} & Other specified types of schizophrenia, in remission \\
\hline 295.10 & Disorganized schizophrenia, unspecified condition & & Unspecified schizophrenia, unspecified condition \\
\hline 295.11 & Disorganized schizophrenia, subchronic condition & \multirow{3}{*}{$\frac{295.91}{295.92}$} & $\begin{array}{l}\text { Unspecitied schizophrenia, unspecitied condition } \\
\text { Unspecified schizophrenia, subchronic condition }\end{array}$ \\
\hline 295.12 & Disorganized schizophrenia, chronic condition & & Unspecified schizophrenia, chronic condition \\
\hline 295.13 & $\begin{array}{l}\text { Disorganized schizophrenia, subchronic condition with acute } \\
\text { exacerbation }\end{array}$ & & $\begin{array}{l}\text { Unspecified schizophrenia, subchronic condition with acute } \\
\text { exacerbation }\end{array}$ \\
\hline 295.14 & $\begin{array}{l}\text { Disorganized schizophrenia, chronic condition with acute } \\
\text { exacerbation }\end{array}$ & 295.94 & $\begin{array}{l}\text { Unspecified schizophrenia, chronic condition with acute } \\
\text { exacerbation }\end{array}$ \\
\hline 295.15 & Disorganized schizophrenia, in remission & \multirow{2}{*}{295.95} & Unspecified schizophrenia, in remission \\
\hline 295.20 & Catatonic schizophrenia, unspecified condition & & Bipolar I disorder, most recent episode (or current) unspecified \\
\hline 295.21 & Catatonic schizophrenia, subchronic condition & \multirow{2}{*}{$\frac{296.00}{296.01}$} & Bipolar I disorder, single manic episode, unspecified \\
\hline 295.22 & Catatonic schizophrenia, chronic condition & & Bipolar I disorder, single manic episode, mild \\
\hline 295.23 & & \multirow{2}{*}{$\frac{296.02}{296.03}$} & \multirow{2}{*}{$\begin{array}{l}\text { Bipolar I disorder, single manic episode, moderate } \\
\text { Bipolar I disorder, single manic episode, severe, without mention of } \\
\text { psychotic behavior }\end{array}$} \\
\hline & exacerbation & & \\
\hline$\frac{295.24}{295.25}$ & $\begin{array}{l}\text { Catatonic schizophrenia, chronic condi } \\
\text { Catatonic schizophrenia, in remission }\end{array}$ & \multirow[t]{2}{*}{296.04} & \multirow{2}{*}{$\begin{array}{l}\text { Bipolar I disorder, single manic episode, severe, specified as with } \\
\text { psychotic behavior }\end{array}$} \\
\hline 295.30 & Paranoid schizophrenia, unspecified condition & & \\
\hline 295.31 & Paranoid schizophrenia, subchronic condition & 296.05 & $\begin{array}{l}\text { Bipolar I disorder, single manic episode, in partial or unspecified } \\
\text { remission }\end{array}$ \\
\hline 295.32 & Paranoid schizophrenia, chronic condition & 296.06 & Bipolar I disorder, single manic episode, in full remission \\
\hline 295.33 & $\begin{array}{l}\text { Paranoid schizophrenia, subchronic condition with acute } \\
\text { exacerbation }\end{array}$ & 296.10 & Manic disorder, recurrent episode, unspecified \\
\hline 295.34 & $\begin{array}{l}\text { exacerbation } \\
\text { Paranoid schizophrenia, chronic condition with acute exacerbation }\end{array}$ & 296.11 & Manic disorder, recurrent episode, mild \\
\hline 295.35 & Paranoid schizophrenia, in remission & 296.12 & Manic disorder, recurrent episode, moderate \\
\hline 295.40 & Schizophreniform disorder, unspecified & 296.13 & $\begin{array}{l}\text { Manic disorder, recurrent episode, severe, without mention of } \\
\text { psychotic behavior }\end{array}$ \\
\hline 295.41 & Schizophreniform disorder, subchronic & 296.14 & Manic disorder, recurrent episode, severe, specified as with \\
\hline 295.42 & Schizophreniform disorder, chronic & 290.14 & \\
\hline 295.43 & Schizophreniform disorder, subchronic with acute exacerbation & 296.15 & \\
\hline 295.44 & Schizophreniform disorder, chronic with acute exacerbation & & remission \\
\hline 295.45 & Schizophreniform disorder, in remission & 296.16 & Manic disorder, recurrent episode, in full remission \\
\hline 295.50 & Latent schizophrenia, unspecified condition & 296.40 & \\
\hline 295.51 & Latent schizophrenia, subchronic condition & & \\
\hline 295.52 & Latent schizophrenia, chronic condition & 296.41 & Bipolar I disorder, most recent episode (or current) manic, mild \\
\hline 295.53 & Latent schizophrenia, subchronic condition with acute exacerbation & 296.42 & Bipolar I disorder, most recent episode (or current) manic, \\
\hline 295.54 & Latent schizophrenia, chronic condition with acute exacerbation & & \\
\hline 295.55 & Latent schizophrenia, in remission & 296.43 & Bipolar I disorder, most recent episode (or current) manic, severe, \\
\hline 295.60 & Schizophrenic disorders, residual type, unspecified & & \\
\hline 295.61 & Schizophrenic disorders, residual type, subchronic & 296.44 & $\begin{array}{l}\text { Bipolar I disorder, most recent episode (or current) manic, severe, } \\
\text { specified as with psychotic behavior }\end{array}$ \\
\hline 295.62 & Schizophrenic disorders, residual type, chronic & 296.45 & \\
\hline 295.63 & $\begin{array}{l}\text { Schizophrenic disorders, residual type, subchronic with acute } \\
\text { exacerbation }\end{array}$ & 290.45 & partial or unspecified remission \\
\hline 295.64 & $\begin{array}{l}\text { Schizophrenic disorders, residual type, chronic with acute } \\
\text { exacerbation }\end{array}$ & 296.46 & $\begin{array}{l}\text { Bipolar I disorder, most recent episode (or current) manic, in full } \\
\text { remission }\end{array}$ \\
\hline 295.65 & $\begin{array}{l}\text { exacerbation } \\
\text { Schizophrenic disorders, residual type, in remission }\end{array}$ & 296.50 & $\begin{array}{l}\text { Bipolar I disorder, most recent episode (or current) depressed, } \\
\text { unspecified }\end{array}$ \\
\hline 295.70 & Schizoaffective disorder, unspecified & 296.51 & Bipolar I disorder, most recent episode (or current) depressed, mild \\
\hline 295.71 & Schizoaffective disorder, subchronic & 296.52 & Bipolar I disorder, most recent episode (or current) depressed, \\
\hline 295.72 & Schizoaffective disorder, chronic & & moderate \\
\hline
\end{tabular}




\section{Association Between Cognitive Function and Health Care Costs 3 Months and 6 Months After Initiating Antidepressant Medication for Depressive Disorders}

\section{APPENDIX C ICD-9-CM Diagnosis Codes for Patient Exclusion (continued)}

\begin{tabular}{|c|c|c|c|}
\hline Code & Description & Code & Description \\
\hline \multirow[t]{2}{*}{296.53} & \multirow{2}{*}{$\begin{array}{l}\text { Bipolar I disorder, most recent episode (or current) depressed, } \\
\text { severe, without mention of psychotic behavior }\end{array}$} & 345.3 & Epileptic grand mal status \\
\hline & & \multirow[t]{2}{*}{345.40} & \multirow{2}{*}{$\begin{array}{l}\text { Localization-related (focal) (partial) epilepsy and epileptic } \\
\text { syndromes with complex partial seizures, without mention of } \\
\text { intractable epilepsy }\end{array}$} \\
\hline 296.54 & $\begin{array}{l}\text { Bipolar I disorder, most recent episode (or current) depressed, } \\
\text { severe, specified as with psychotic behavior }\end{array}$ & & \\
\hline 296.55 & $\begin{array}{l}\text { Bipolar I disorder, most recent episode (or current) depressed, in } \\
\text { partial or unspecified remission }\end{array}$ & 345.41 & $\begin{array}{l}\text { Localization-related (focal) (partial) epilepsy and epileptic } \\
\text { syndromes with complex partial seizures, with intractable epilepsy }\end{array}$ \\
\hline 296.56 & $\begin{array}{l}\text { Bipolar I disorder, most recent episode (or current) depressed, in } \\
\text { full remission }\end{array}$ & 345.50 & $\begin{array}{l}\text { Localization-related (focal) (partial) epilepsy and epileptic } \\
\text { syndromes with simple partial seizures, without mention of }\end{array}$ \\
\hline \multirow[t]{2}{*}{296.60} & \multirow{2}{*}{$\begin{array}{l}\text { Bipolar I disorder, most recent episode (or current) mixed, } \\
\text { unspecified }\end{array}$} & & \\
\hline & & \multirow[t]{2}{*}{345.51} & \multirow{2}{*}{$\begin{array}{l}\text { Localization-related (focal) (partial) epilepsy and epileptic } \\
\text { syndromes with simple partial seizures, with intractable epilepsy }\end{array}$} \\
\hline 296.61 & Bipolar I disorder, most recent episode (or current) mixed, mild & & \\
\hline \multirow[t]{2}{*}{296.62} & \multirow{2}{*}{$\begin{array}{l}\text { Bipolar I disorder, most recent episode (or current) mixed, } \\
\text { moderate }\end{array}$} & 345.60 & Infantile spasms without mention of intractable epilepsy \\
\hline & & 345.61 & Infantile spasms with intractable epilepsy \\
\hline \multirow[t]{2}{*}{296.63} & \multirow{2}{*}{$\begin{array}{l}\text { Bipolar I disorder, most recent episode (or current) mixed, severe, } \\
\text { without mention of psychotic behavior }\end{array}$} & 345.70 & Epilepsia partialis continua without mention of intractable epilepsy \\
\hline & & 345.71 & Epilepsia partialis continua with intractable epilepsy \\
\hline 296.64 & $\begin{array}{l}\text { Bipolar I disorder, most recent episode (or current) mixed, severe, } \\
\text { specified as with psychotic behavior }\end{array}$ & 345.80 & $\begin{array}{l}\text { Other forms of epilepsy and recurrent seizures, without mention of } \\
\text { intractable epilepsy }\end{array}$ \\
\hline 296.65 & $\begin{array}{l}\text { Bipolar I disorder, most recent episode (or current) mixed, in } \\
\text { partial or unspecified remission }\end{array}$ & 345.81 & $\begin{array}{l}\text { Other forms of epilepsy and recurrent seizures, with intractable } \\
\text { epilepsy }\end{array}$ \\
\hline 296.66 & \multirow{2}{*}{$\begin{array}{l}\text { Bipolar I disorder, most recent episode (or current) mixed, in full } \\
\text { remission }\end{array}$} & 345.90 & Unspecified epilepsy without mention of intractable epilepsy \\
\hline 296.80 & & 345.91 & Unspecified epilepsy with intractable epilepsy \\
\hline 296.81 & Atypical manic disorder & 649.40 & $\begin{array}{l}\text { Epilepsy complicating pregnancy, childbirth, or the puerperium, } \\
\text { unspecified as to episode of care or not applicable }\end{array}$ \\
\hline 296.89 & Other and unspecified bipolar disorders & \multirow{2}{*}{649.41} & \multirow{2}{*}{$\begin{array}{l}\text { Epilepsy complicating pregnancy, childbirth, or the puerperium, } \\
\text { delivered, with or without mention of antepartum condition }\end{array}$} \\
\hline \multirow[t]{2}{*}{345.00} & \multirow{2}{*}{$\begin{array}{l}\text { Generalized nonconvulsive epilepsy without mention of intractable } \\
\text { epilepsy }\end{array}$} & & \\
\hline & & 649.42 & $\begin{array}{l}\text { Epilepsy complicating pregnancy, childbirth, or the puerperium, } \\
\text { delivered, with mention of postpartum complication }\end{array}$ \\
\hline 345.10 & & 649.43 & \\
\hline & & 049.45 & $\begin{array}{l}\text { Epllepsy complicating pregnancy, childbirth, or the puerperium, } \\
\text { antepartum condition or complication }\end{array}$ \\
\hline 345.11 & Generalized convulsive epilepsy with intractable epilepsy & \multirow[t]{2}{*}{649.44} & \multirow{2}{*}{$\begin{array}{l}\text { Epilepsy complicating pregnancy, childbirth, or the puerperium, } \\
\text { postpartum condition or complication }\end{array}$} \\
\hline 345.2 & Epileptic petit mal status & & \\
\hline
\end{tabular}




\section{Association Between Cognitive Function and Health Care Costs 3 Months and 6 Months After Initiating Antidepressant Medication for Depressive Disorders}

\section{APPENDIX D List of Medications for Patient Exclusion}

\section{Condition}

Schizophrenia
Generic Name (Brand Name)

amitriptyline/perphenazine (Triavil)

amitriptyline/perphenazine (Etrafon)

chlorpromazine (Thorazine)

fluphenazine (Prolixin)

fluphenazine (Permitil)

haloperidol (Haldol)

loxapine (Loxitane)

mesoridazine (Serentil)

molindone (Moban)

perphenazine (Trilafon)

pimozide (Orap)

thioridazine (Mellaril)

thiothixene (Navane)

trifluoperazine (Stelazine)

triflupromazine (Vesprin)

aripiprazole (Abilify)

asenapine (Saphris)

clozapine (Clozaril)

clozapine (Fazaclo)

iloperidone (Fanapt)

lurasidone (Latuda)

olanzapine (Zyprexa)

olanzapine/fluoxetine (Symbyax)

paliperidone (Invega)

quetiapine (Seroquel)

risperidone (Risperdal)

ziprasidone (Geodon)

lithium (Eskalith)

lithium (Lithobid)

lithium (Lithotabs)

Bipolar disorder/ epilepsy carbamazepine (Tegretol)

carbamazepine (Equetro)

divalproex/valproate/valproic acid (Depacon)

divalproex/valproate/valproic acid (Depakene)

divalproex/valproate/valproic acid (Depakote)

divalproex/valproate/valproic acid (Depakote ER)

divalproex/valproate/valproic acid (Stavzor)

ethotoin (Peganone)

fosphenytoin sodium (Cerebyx)

mephenytoin (Mesantoin)

mephobarbital (Mebaral)

methsuximide (Celontin)

phenobarbital (Solfoton)

phenobarbital sodium (Luminal)

phensuximide (Milontin)

phenytoin (Dilantin)

phenytoin sodium (Dilantin)

phenytoin sodium (Phenytek)

phenytoin sodium extended (Dilantin)

\section{Condition}

Bipolar disorder/

epilepsy

Alzheimer's disease

Huntington's disease

Parkinson's disease
Generic Name (Brand Name)

primidone (Mysoline)

gabapentin (Neurontin)

ethosuximide (Zarontin)

ezogabine (Potiga)

felbamate (Felbatol)

lacosamide (Vimpat)

lamotrigine (Lamictal)

lamotrigine (Lamictal XR)

levetiracetam (Keppra)

levetiracetam (Keppra XR)

oxcarbazepine (Trileptal)

rufinamide (Banzel)

tiagabine (Gabitril)

vigabatrin (Sabril)

zonisamide (Zonegran)

donepezil (Aricept)

tacrine (Cogenex)

rivastigmine (Exelon)

galantamine (Razadyne)

galantamine (Razadyne ER)

memantine (Namenda) and XR

memantine (Namenda XR)

interferon $\beta$-lb (Betaseron)

interferon $\beta$-la (Avonex)

glatiramer acetate (Copaxone)

interferon $\beta$-la (Rebif)

natalizumab (Tysabri)

interferon $\beta$-lb (Extavia)

dalfampridine (Ampyra)

fingolimod (Gilenya)

tetrabenazine (Xenazine)

carbidoba/levodopa (Parcopa)

carbidoba/levodopa (Sinemet)

carbidoba/levodopa (Sinemet CR)

rasagiline (Azilect)

selegiline (Eldepryl)

selegiline (Zelapar)

apomorphine (Apokyn)

pramipexole (Mirapex)

pramipexole (Mirapex ER)

ropinirole (Requip)

ropinirole (Requip XL)

rotigotine (Neupro)

entacapone (Comtan)

tolcapone (Tasmar)

benztropine (Cogentin)

trihexyphenidyl (Artane)

biperiden (Akineton)

amantadine (Symmetrel)

carbidopa/levodopa/entacapone (Stalevo)

$C R=$ controlled release; $E R=$ extended release; $X L=$ extended release; $X R=$ extended release 


\section{Association Between Cognitive Function and Health Care Costs 3 Months and 6 Months After Initiating Antidepressant Medication for Depressive Disorders}

\begin{tabular}{|c|c|}
\hline APPEND & $\begin{array}{l}\text { Other Medications Related to } \\
\text { Depressive Disorder Included } \\
\text { in the Cost of Depression }\end{array}$ \\
\hline Drug Class & Generic Name (Brand Name) \\
\hline \multirow[t]{3}{*}{ Antipsychotics } & aripiprazole (Abilify) \\
\hline & olanzapine/fluoxetine (Symbyax) \\
\hline & quetiapine (Seroquel, Seroquel XR) \\
\hline \multirow[t]{9}{*}{ Anxiolytics } & alprazolam (Xanax) \\
\hline & buspirone (Buspar) \\
\hline & diazepam (Valium) \\
\hline & lorazepam (Ativan) \\
\hline & chlordiazepoxide (Librium) \\
\hline & meprobamate (Equanil) \\
\hline & clonazepam (Klonopin) \\
\hline & oxazepam (Serax) \\
\hline & clorazepate (Gen-xene) \\
\hline \multirow[t]{7}{*}{ Hypnotics } & chloral hydrate (Somnote) \\
\hline & eszopiclone (Lunesta) \\
\hline & ramelteon (Rozerem) \\
\hline & temazepam (Restoril) \\
\hline & triazolam (Halcion) \\
\hline & zaleplon (Sonata) \\
\hline & zolpidem (Ambien) \\
\hline
\end{tabular}

APPENDIX F Analysis of All-Cause and Depressive Disorder-Related Cost Ratios by Follow-up Period

\begin{tabular}{|c|c|c|c|c|c|c|c|c|c|c|c|c|}
\hline \multirow[b]{3}{*}{ Independent Variables } & \multicolumn{6}{|c|}{ All-Cause Costs } & \multicolumn{6}{|c|}{ Depressive Disorder-Related Costs } \\
\hline & \multicolumn{3}{|c|}{ 3-Month Follow-up ${ }^{a}$} & \multicolumn{3}{|c|}{ 6-Month Follow-up ${ }^{\mathrm{b}}$} & \multicolumn{3}{|c|}{ 3-Month Follow-up ${ }^{a}$} & \multicolumn{3}{|c|}{ 6-Month Follow-up ${ }^{b}$} \\
\hline & $\begin{array}{l}\text { Cost } \\
\text { Ratio }\end{array}$ & $95 \%$ CI & $\begin{array}{c}P \\
\text { Value }\end{array}$ & $\begin{array}{l}\text { Cost } \\
\text { Ratio }\end{array}$ & $95 \%$ CI & $\begin{array}{c}P \\
\text { Value }\end{array}$ & $\begin{array}{l}\text { Cost } \\
\text { Ratio }\end{array}$ & $95 \%$ CI & $\begin{array}{c}P \\
\text { Value }\end{array}$ & $\begin{array}{l}\text { Cost } \\
\text { Ratio }\end{array}$ & $95 \%$ CI & $\begin{array}{c}P \\
\text { Value }\end{array}$ \\
\hline Intercept & $2,128.94$ & $\begin{array}{l}1,201.05- \\
3,773.69\end{array}$ & $<0.001$ & $2,275.25$ & $\begin{array}{l}1,393.29- \\
3,715.48\end{array}$ & $<0.001$ & $1,705.35$ & $\begin{array}{c}878.78- \\
3,309.36\end{array}$ & $<0.001$ & 894.46 & $\begin{array}{l}503.99- \\
1,587.43\end{array}$ & $<0.001$ \\
\hline $\begin{array}{l}\text { Patient has cognitive } \\
\text { dysfunction }\end{array}$ & 1.53 & $1.17-2.02$ & 0.002 & 1.30 & $1.02-1.66$ & 0.034 & 1.77 & $1.30-2.41$ & $<0.001$ & 1.30 & $0.98-1.72$ & 0.071 \\
\hline $\begin{array}{l}\text { Patient has any mental } \\
\text { health-related AHRQ } \\
\text { comorbidityc }\end{array}$ & 0.83 & $0.63-1.10$ & 0.196 & 1.00 & $0.79-1.27$ & 0.980 & 1.11 & $0.80-1.54$ & 0.542 & 1.17 & $0.89-1.54$ & 0.268 \\
\hline $\begin{array}{l}\text { Baseline Quan-Charlson } \\
\text { score >0 }\end{array}$ & 3.76 & $2.51-5.63$ & $<0.001$ & 4.51 & $3.20-6.36$ & $<0.001$ & 3.08 & $1.92-4.93$ & $<0.001$ & 6.30 & $4.19-9.47$ & $<0.001$ \\
\hline $\begin{array}{l}\text { Patient-reported education } \\
\text { "some college" or less }{ }^{\mathrm{d}}\end{array}$ & 1.02 & $0.78-1.34$ & 0.877 & 0.96 & $0.75-1.23$ & 0.755 & 0.68 & $0.49-0.94$ & 0.018 & 0.80 & $0.61-1.07$ & 0.129 \\
\hline White race & 0.94 & $0.58-1.54$ & 0.819 & 0.85 & $0.54-1.32$ & 0.468 & 0.53 & $0.30-0.94$ & 0.029 & 1.53 & $0.89-2.64$ & 0.128 \\
\hline Male gender & 1.15 & $0.83-1.60$ & 0.399 & 1.28 & $0.95-1.71$ & 0.100 & 0.42 & $0.30-0.61$ & $<0.001$ & 0.68 & $0.48-0.95$ & 0.026 \\
\hline Age (continuous) & 0.99 & $0.98-1.00$ & 0.149 & 1.01 & $1.00-1.02$ & 0.231 & 0.96 & $0.95-0.97$ & $<0.001$ & 0.98 & $0.97-0.99$ & 0.001 \\
\hline Full-time employment ${ }^{\mathrm{f}}$ & 1.40 & $1.06-1.85$ & 0.017 & 1.00 & $0.78-1.28$ & 0.987 & 2.02 & $1.47-2.78$ & $<0.001$ & 1.47 & $1.09-1.98$ & 0.012 \\
\hline
\end{tabular}

${ }^{a}$ Number of observations used: 559 out of an original population of 563.

${ }^{b}$ Number of observations used: 555 out of an original population of 559 .

'Agency for Healthcare Research and Quality Clinical Classification Software identification.

¿Patient's highest education level achieved (less than high school, some high school, high school or equivalent [e.g., GED], some college but no degree, 2-year degree [community or technical], college graduate, graduate school).

'Patient's self-identification as American Indian, Alaska Native, Asian, Native Hawaiian or Pacific Islander, Black or African American, White, Other race (reporting more than 1 entry was possible).

fPatient-reported employment status (employed full time, employed part time, homemaker, retired, unemployed, other). $A H R Q=$ Agency for Healthcare Research and Quality; $C I=$ confidence interval; GED = general education development. 